\title{
Random Visitor: Defense against Identity Attacks in P2P Networks*
}

\author{
Jabeom GU ${ }^{\dagger a)}$, Jaehoon NAH ${ }^{\dagger}$, Hyeokchan KWON ${ }^{\dagger}$, Jongsoo JANG $^{\dagger}$, Nonmembers, \\ and Sehyun PARK ${ }^{\dagger \dagger}$, Member
}

SUMMARY Various advantages of cooperative peer-to-peer networks are strongly counterbalanced by the open nature of a distributed, serverless network. In such networks, it is relatively easy for an attacker to launch various attacks such as misrouting, corrupting, or dropping messages as a result of a successful identifier forgery. The impact of an identifier forgery is particularly severe because the whole network can be compromised by attacks such as Sybil or Eclipse. In this paper, we present an identifier authentication mechanism called random visitor, which uses one or more randomly selected peers as delegates of identity proof. Our scheme uses identity-based cryptography and identity ownership proof mechanisms collectively to create multiple, cryptographically protected indirect bindings between two peers, instantly when needed, through the delegates. Because of these bindings, an attacker cannot achieve an identifier forgery related attack against interacting peers without breaking the bindings. Therefore, our mechanism limits the possibility of identifier forgery attacks efficiently by disabling an attacker's ability to break the binding. The design rationale and framework details are presented. A security analysis shows that our scheme is strong enough against identifier related attacks and that the strength increases if there are many peers (more than several thousand) in the network.

key words: peer-to-peer, overlay network, security, identifier authentication, random visitor, identity-based cryptography

\section{Introduction}

Peer-to-peer (P2P) overlay networking is generally defined, perhaps a little simplistically, as the study of sharing network resources between heterogeneous, massive computing nodes in a distributed and collaborative manner. It is called an overlay because the majority of the functions that comprise such a network are performed at the application layer. Resource sharing has may variations such as allocating tasks as in SETI@home [1] and FOLDING@home [2]; exchanging information as in Jabber [3] and Groove [4]; and distributing files as in Gnutella [5], KaZaa [6], and Napster [7].

Manuscript received April 9, 2007.

Manuscript revised October 18, 2007.

${ }^{\dagger}$ The authors are with the Electronics and Telecommunications Research Institute (ETRI), Daejeon 305-700, Korea.

${ }^{\dagger \dagger}$ The author is with the faculty of Chung-Ang University, Seoul 156-756, Korea.

*Preliminary version of this work was presented at WISA 2006. This research was partly supported by the MIC (Ministry of Information and Communication), Korea, under the HNRC ITRC (Information Technology Research Center) support program supervised by the IITA (Institute of Information Technology Assessment), the Chung-Ang University Excellent Researcher Grant in 2007, and the IT R\&D program of MIC/IITA [2005-S-09003, Development of P2P Network Security Technology based on Wired/Wireless IPv6 Network].

a)E-mail: gjb@etri.re.kr

DOI: $10.1093 /$ ietisy/e91-d.4.1058
This type of networking has many unique properties, including a lack of hierarchical organization or centralized control for management support and a dynamic membership allowing people to join and leave the network freely.

P2P networking itself is not a new concept, but since the popularity and attention given to Napster [7] in 1999, the increasing activity in file sharing communities has served as a good testbed for the realization of a distributed and collaborative resource sharing idea. The development of the most recent VoIP application, called Skype [8], is remarkable in the sense that it is intrinsically based on the well-known P2P framework, namely the KaZaa network [6], [9]. This avalanche of successful implementation and deployment of applications has already shown an encouraging potential of $\mathrm{P} 2 \mathrm{P}$, and the outlook for this new computing paradigm seems promising, not only for academia but also for industry.

But constructing such a distributed and collaborative resource sharing network requires more attention. When dealing with large sets of shared resources and collaborating peers, the first and most basic problem is representing resources and peers uniquely in a network and locating them with accuracy (namely, with a high level of trust). Many recent solutions to P2P resource discovery assume the following:

A1) the existence of globally unique identifiers;

A2) the existence of an algorithm that allocates uniform, randomly distributed identifiers; and

A3) that participating nodes are cooperative and trustworthy in generating and handling their identifiers.

However, these assumptions are not easy to achieve in an adversarial environment like a P2P network, especially when there are no centralized management functions.

Furthermore, any participating node should be able to examine and determine the truthfulness of the claims about the identifier made by another node that it encounters in a global-scale, Internet-sized P2P network. This is undoubtedly challenged by the open nature of the overlays. More precisely, the network will comprise heterogeneous participants with different operational contexts including personal interests, resource plans, security considerations, and so on. Therefore, we cannot always expect friendly collaboration between distributed parties.

Castro et al. [10] identified the following three major requirements to ensure security of the overlay: $R l$ ) secure 
identifier assignment mechanism, $R 2$ ) secure routing table maintenance, and R3) secure message forwarding. When these requirements are not addressed, any overlay is vulnerable to insider attacks such as misrouted, corrupted, or dropped messages and routing information triggered by those uncooperative, untrusted nodes. The aforementioned P2P overlay networks fail to address these attacks because requirements $R I-R 3$ are rarely or even never considered.

As a result, distributed networking with no centralized management server has a great deal of vulnerability such as misrouted, corrupted, or dropped communication messages and routing information due to a potentially successful identifier forgery. In unmanaged P2P networks, the impact of an identifier forgery is particularly severe because the whole network can be compromised by a single attacker through attacks like Sybil or Eclipse [10], [11]. When there are indications of the possibility of such attacks, it is difficult to validate the trustworthiness of a claimed identifier. These attacks are easy to be exploited because of the open nature of the P2P overlay networks. We refer to the assumptions $A 1-A 3$ and requirements $R 1-R 3$ and define related security threats and vulnerabilities collectively as an identifier authentication problem.

In this paper, we attempt to fulfill the requirements $R I-$ $R 3$ and propose a solution for the identifier authentication problem in large-sized, serverless $\mathrm{P} 2 \mathrm{P}$ overlay networks using identity-based cryptography [12]-[15] and identity ownership proof [16]-[18]. In our scheme, these mechanisms are collectively used to create multiple, cryptographically protected indirect trust bindings between two peers, instantly when needed.

More precisely, a $\mathrm{P} 2 \mathrm{P}$ node creates one or more cryptographic 'trust bindings' with remote peer(s) easily and instantly without any help from a centralized manager or server. The node can interpret the cryptographic binding as its trust upon a remote peer(s) identifier because the scheme combines the peer's identifier to the generated trust binding. Because of this binding, an attacker cannot do much harm to the peers (like misrouting, corrupting, or dropping communication data transferred between two peers) as a result of an identifier forgery. Therefore, our mechanism limits the possibility of identifier forgery by disabling an attacker to break the binding. A security analysis shows that our scheme is strong enough against identifier related attacks and that the strength increases if there are many peers (more than several thousand) in the network.

It is assumed that there is no management server in a P2P network. It is further assumed that we cannot rely on such user authentication mechanisms as Public Key Infrastructure (PKI) or any server-based access control schemes. In our approach, a peer node (or an initiator) tries to detect the existence of any identifier-related attacks and check the validity of the identifier of a remote peer (or a target). To do this, the initiator creates an indirect binding using a randomly chosen delegate, called a random visitor, who delivers a credential to the target, with which the target can prove sincerity, ownership, and moderate exclusiveness of its identifier.

The remainder of this paper is organized as follows. Section 2 describes the background and related work. Section 3 presents the overall concept of our scheme. Section 4 presents framework details of our scheme. Section 5 presents a security analysis of the proposed scheme. A prototype implementation and measurement are presented in Sect. 6. Section 7 concludes the paper.

The paper is an extended and revised version of [19] presented at WISA 2006.

\section{Preliminary}

\subsection{Identifier in P2P Networks}

We consider a dynamic, serverless $\mathrm{P} 2 \mathrm{P}$ network with $N$ participating nodes. The number of nodes $N$ may change over time because nodes may join or leave the network. Each node can freely generate one or more globally unique identifier $^{\dagger} i d$ using a generation function $f$. Input of the generation function is dependent on the specific implementation details of the network.

Closely related to the generation function are an identifier allocation function and a lookup function. While numerous specific details may differ, the P2P overlay generally manages identifier allocation and identifier lookup functions, i.e., $F_{A}$ and $F_{R}$. The allocation function assigns a generated identifier to a single node (called a root or target of the identifier) such that the IP address of the root can be searched using the lookup function. The lookup function routes the query for an identifier, initiated by a peer node, through the routing path until it gets to the root. Gnutella's unstructured overlay uses no allocation function, so there exists some inefficiency in its lookup function. Structured overlays like CAN, Chord, Pastry, and Tapestry use a Distributed Hash Table (DHT) to construct efficient allocation and lookup functions [20]. Notations that will be used throughout this paper are presented in Table 1.

In some distributed approaches (without any central or hierarchical support) such as Gnutella [5], natural names (for example, filenames or attributes) are used for this purpose. This type of approach is called an unstructured overlay because the overall architecture is loosely controlled and resources are distributed randomly all over the network. In other distributed cases like CAN [21], Chord [22], Pastry [23], and Tapestry [24], tailored bit strings are used to achieve maximum efficiency in routing and discovery. This type of approach is also called a structured overlay. When the network has some hierarchy, as in the case of a wellknown JXTA framework [25], [26], a dedicated mechanism is employed to ensure global uniqueness.

\footnotetext{
†' In general P2P networks, identifiers are used extensively to represent and distinguish various entities (peer identifier or user identifier) and shared resources (resource identifier). Throughout this paper, we use identifier to refer both entity identifier and resource identifier.
} 
Table 1 Notations.

\begin{tabular}{|l|l|}
\hline$f$ & Identifier generation function \\
$v$ & Identifier verification function \\
$F_{A}$ & Identifier allocation function \\
$F_{R}$ & Routing and lookup function \\
$F_{p r i}$ & IBC Private key generation function \\
$F_{R V}$ & Proposed random visitor scheme \\
$\mathcal{P}$ & Set of nodes participating in a P2P network \\
$\mathcal{I}$ & Identifier space \\
$\mathcal{T}$ & Time space \\
$\mathcal{D}$ & delegate space \\
$\mathcal{R}$ & Set of resources in the network \\
$t_{s}$ & Sojourn time of random visitor \\
$\mathrm{ID}_{\mathrm{k}}$ & Static, globally unique identifier of node $k$ \\
$\mathrm{IP}$ & IP address of node $k$ \\
$a i d_{k}$ & Auxiliary identifier of node $k$ in the overlay network \\
$N_{i}$ & Nonce generated by initiator \\
$N_{t}$ & Nonce generated by target \\
$N_{v}$ & Nonce generated by initiator to verify peer identifier \\
$K_{k}^{u}$ & RSA Public key of node $k$ \\
$K_{k}^{r}$ & RSA Private key of node $k$ \\
$S_{K_{k}^{r}}^{r}(m)$ & Public key signature, signing message $m$ using node $k$ 's \\
$E_{i d_{k}}(m)$ & private key, $K_{k}^{r}$ \\
$D_{i d_{k}}(c)$ & IBC encryption function, encrypting message $m$ using \\
$S_{i d_{k}}(m)$ & identifier of node $k, i d_{k}$ \\
$\epsilon$ & IBC decryption function, decrypting cipher text $c$ using \\
$p r$ & identifier of node $k, i d_{k}$ \\
$H$ & IBC signature function, signing message $m$ using identifier \\
\hline & of node $k, i d_{k}$ \\
\hline
\end{tabular}

\subsection{Identity Attacks and Solutions}

Although the dependency of the overlay onto the validity of the identifier is critical, recently proposed overlays rarely or even never consider authenticity verification. As a result, it is easy for an attacker to control the identifier space such that he can launch various identifier-related attacks such as misrouting, corrupting, or dropping messages. Such malicious control of identifier space is known as Sybil and Eclipse attacks [10], [11]. These attacks are easy to be exploited because of the open nature of the P2P overlay networks.

J. Douceur[11] coined the term Sybil attack and showed that in an environment where any entity can generate an identifier using $f$, an attacker can also generate as many identifiers as needed to forge the allocation or lookup function, $F_{A}$ or $F_{R}$. When a reasonably large subset of the identifier space $\mathcal{I}$ is controlled by an attacker, it can launch an initiator-based or a target-based attack [27]. These two attacks are illustrated in Fig. 1.

In an initiator (or source) based attack, an attacker selects a victim and places several first-hop neighbors near the victim so that he can fill the routing table of the victim and forge $F_{R}$. This attack is also known as an Eclipse attack [10], [28], [29]. This attack is possible because each node collects routing information directly from its 1st-hop neighbors during the bootstrapping procedure [30]. This is shown in Fig. 1 (a). For example, let a node in the matrix be identified by its coordinate such that the initiator is lo-

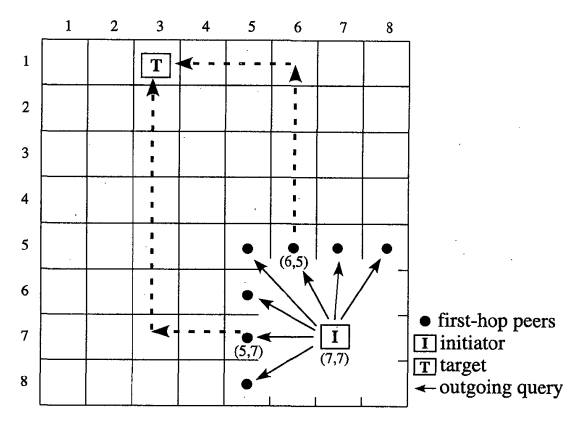

(a)

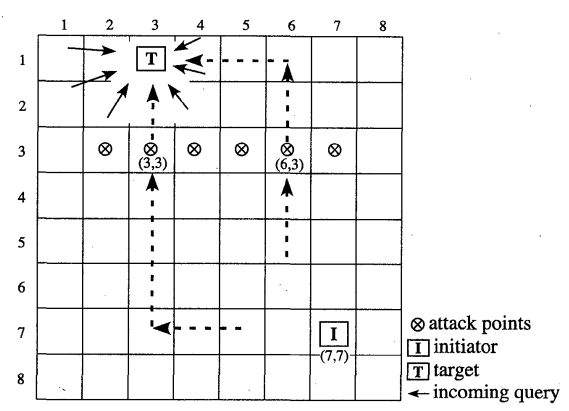

(b)

Fig. 1 Two-dimensional illustration of initiator-based and target-based attacks: (a) initiator-based attack and (b) target-based attack.

cated at $(7,7)$ and the target is located at $(3,1)$. In this figure, two dashed lines indicate two routing paths from the initiator (I) to the target (T). Thus, among the 1st-hop nodes, only nodes $(6,5)$ and $(5,7)$ are responsible to deliver an initiator's message to the target. A query to $(6,5)$ will follow $(6,5) \rightarrow(6,4) \rightarrow(6,3) \rightarrow(6,2) \rightarrow(6,1) \rightarrow(5,1) \rightarrow(4,1) \rightarrow(T)$. Therefore, if an attacker can forge the identity of nodes $(6,5)$ and $(7,5)$, he can successfully attack the communication between the initiator and the target. To isolate the initiator from the network, the Eclipse attacker generally forges all first-hop neighbors. Because there are only a few first-hop neighbors, the computational cost to mount such an attack is relatively low.

In a target-based attack, an attacker places forged identifiers near a target node or on the routing path so that the lookup procedure can be forged or hijacked. When these identifier related attacks are successfully launched, the victim can be partitioned (or isolated) from the overlay network, or can even be hijacked to a fake network [31]. For example in Fig. 1 (b), identifiers of the nodes $(2,3),(3,3)$, $(4,3),(5,3),(6,3)$, and $(7,3)$ are forged. Because the two routing paths from the initiator to the target are in the range of those compromised nodes, an attacker can successfully manipulate transactions between the two nodes. Note that the attack is successful only when the attack range covers the routing path. Therefore, the attack is greatly efficient if the compromised nodes are close to the target.

We use a packet relay network as an example to demonstrate the effect of a type of attack in the GloMoSim simu- 


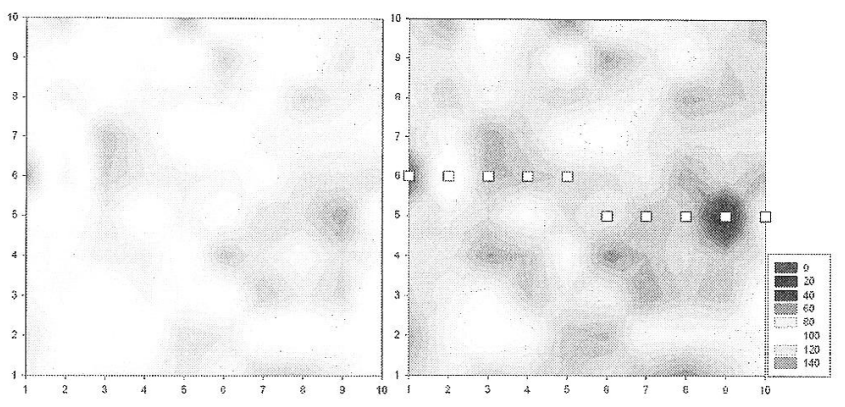

Fig. 2 Spectrum of intensity indicating number of successfully delivered packets at each peer indexed from $(1,1)$ to $(10,10)$. (a) No identifier attacks. (b) Ten attacked identifiers (attacked peers are marked with small squares).

lator [32]. For simplicity, we have only considered a dropping attack. One hundred peers are placed randomly in a two-dimensional area, and their indexes are used as identifiers. To clarify the result, we have shown in Fig. 2 a spectrumized representation of the intensity (number of packet) at each point. Figure 2 (a) shows the number of successfully delivered packets at each peer in normal conditions without any identifier forgery attack. Figure 2 (b) shows when some identifiers are forged (attacked peers are marked with small squares).

Assuming that the peers are statistically identical, the simulation result shows that the forged identifiers can be used collectively to degrade the robustness of the entire network. Figure 3 also shows the number of packets successfully received at each peer. Linear regression lines shown as dashed lines in Fig. 3 were fitted to determine the average number of received packets at each peer. It shows that $10 \%$ of the packets are dropped throughout the network.

Until now, various studies have been performed to defend against such identity attacks. A signed existence proof mechanism [27] uses an estimation of the namespace density, which indicates a possible attack when the density is greater than a threshold. If a node detects a suspicious identity, then it requests a signed proof material which is stored in randomly selected multiple nodes, called proof managers, to provide a proof of existence that the identifier really belongs to a node in the network.

In a cooperative admission control scheme [33], a node wishing to join the network should solve multiple puzzles presented by multiple nodes that reside in an adaptively constructed node set. This scheme limits the rate at which an attacker can create forged identifiers to launch a Sybil attack.

A circumvention mechanism proposed in [34] exploits a tailored routing strategy, which alleviates the effect of the Sybil attack. The mechanism uses multiple lookups performed simultaneously through a diverse set of nodes, thus some of the requests can reach the target.

A self-registration scheme [35] binds an identifier to the IP address. If a node tries to join the network, then it should verify its identity to multiple nodes. Similarly, S. Čapkun et al. [36] present public-key-based and symmetric-key-based security association mechanism, in which the IP address and public key of a node are hashed

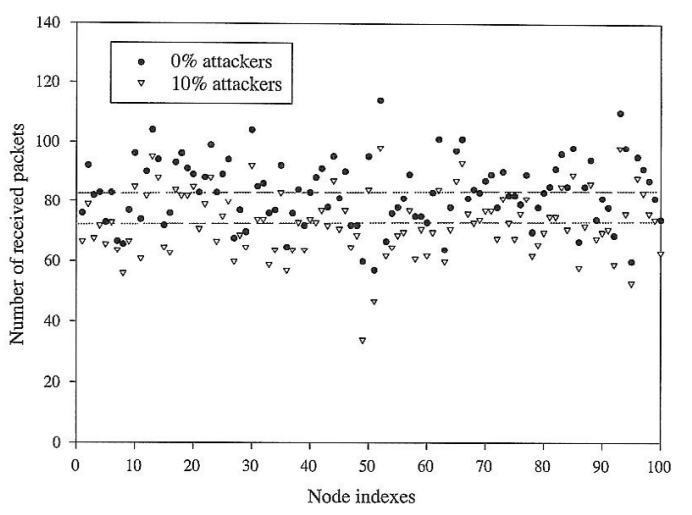

Fig.3 Number of successfully delivered packets at each peer. Two linear regression lines indicate that an average of $10 \%$ packets are dropped throughout the network when 10\% of the identifiers are forged (attack points are marked with little squares).

to produce a credential that can be used for further authentication.

Singh et al. [29] proposed a bounding indegree and outdegree of an overlay node within a threshold. Because the Sybil or Eclipse attack cause the in or out degree to be relatively higher than the ordinary nodes, the nodes should select their neighbor such that the node degree does not exceed the threshold. An audit trailing is used to keep track of the node degree.

Sit and Morris [31] summarized these approaches and presented design principles for a potential defense against identity threats, some of which are listed below:

P1) Define verifiable system invariants.

Q2) Allow the querier to observe the lookup progress.

P3) Assign keys to nodes in a verifiable way.

P4) Cross-check routing tables using random queries.

These principles occur in several researches dealing with identity protection, and some of them have been partly addressed by previous work. For example, [35] and [36] addresses $\mathcal{P} 1$ by introducing such invariants as an IP address into the generation of an identifier. Auditing in [29] is possibly a way of observing the lookup progress of $P 2$. Principle $P 3$ is partly addressed by [27] and [33]. That is, only one who has admission can join the network with a given identifier. The multiple querying scheme in [34] is a way of cross-checking the routing table. If some or all of the queries fails, then there is an on-going identity attack.

\subsection{Problems with Previous Approaches}

In this paper, we contend that "when an identifier generation function $f$ is public, any node that is 'sincere' enough to follow the restriction posed by $f$ (some cryptographic puzzles [33], for example) can join the network successfully, and observation alone cannot distinguish an attacker from a decent user."

The indegree and outdegree observation in [29] cannot be applied when an attacker controls extremely few, but critical, identifiers. Therefore, most of the recent approaches 
to defend against identity attacks such as Sybil and Eclipse rely on an admission control by which identity verification function $v$ tests the sincerity of the node. We refer to the mechanisms as a sincerity proof scheme. Or to put it simply, this scheme accepts anyone who appears to be sincere.

As criticized in [10], however, the sincerity proof scheme is limited by itself when the scheme is applied in a heterogeneous environment, where participating nodes have a different computing resource. Furthermore, Douceur [11] has shown that "Although a Sybil attack can be limited to some degree by the sincerity proof scheme, the attacker can still have an opportunity to generate as many identities as he needs; and the amount has lower bound $\rho$, which is the ratio of the computing resource of an attacker to the resource of a decent entity who has minimal capability."

\section{Our Approach}

\subsection{Basic Concepts}

In this subsection, we present our underlying philosophy that guided us to realize the design principles of $P_{1}-P_{4}$. As discussed in the previous subsection, it is evident that we can have moderate invariant ( $\mathscr{P} 1)$ by binding an IP address to an identifier. But the other three principles need further explanation.

Suppose that an attacker has forged a large enough number of identifiers and has placed several 'slave' nodes along the routing path from an initiator to a target. This is illustrated in Fig. 4. In the figure, the crossed circles indicates adequate attack points. In the example scenario of Fig. 4 (a), an attacker is trying to re-route all packets addressed to the target. Therefore,

Lemma 1. if those slaves can re-route packets from the initiator to the target, then the attack is considered successful.

From the second example in Fig. 4 (b),

Lemma 2. when there are several alternate routing paths from the initiator to the target, the attack is considered successful only when the attacker can forge all such paths.

Considering Lemma 1 and 2, our design is mostly mo-

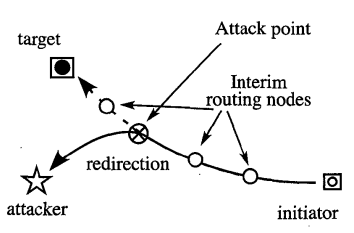

(a)

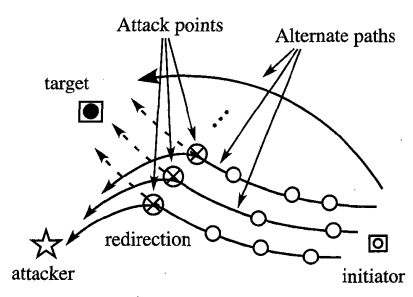

(b)
Fig. 4 Example of target-based attacks in multi-hop relay-based communications (a) when there is only one path and (b) when there are multiple paths. tivated from the following:

- Blinding attacker: first and most evident from the scenario in Fig. 4 (a) is that fixed nodes can easily be a target of an attack. Thus, our solution is to create a 'blinding' situation so that an attacker cannot premeditate an action. This is realized by ensuring randomness in determining the validity of a peer identifier.

- Moderate trust: in this paper, we contend that a hardening approach, even a weak one, is enough for P2P overlay networks to make attacking a demanding job. Actually, in many distributed P2P 'open environments,' using an authentication mechanism like PKI is too much of a luxury, not to mention technically infeasible. We achieve a high level of trust by introducing a randomly selected delegate and binding three tiers 〈initiator-delegate-target ) using a moderate, efficient mechanism based on identity-based cryptography.

- Decreasing possibility of a successful attack: a simple way to do this is to use parallel redundancy assuming that attack events against peers are mutually independent. As illustrated in Fig. 4 (b), repeated application of our scheme will naturally provide this feature.

Figure 5 shows a comparison of the trust binding of our scheme with that of the Public Key Infrastructure (PKI). The PKI is a collection of technical and organisational facilities that provides a trusted third party to attest to the key authentication problem - the identity of the public key holder should be bound to the public key of that holder. In PKI, a trustworthy third party (called a certificate authority or certification authority (CA)) is arranged to publish trust materials, called certificates, which can be used to validate the binding between a user and his or her public key. Thus trust

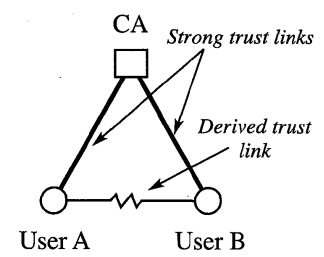

(a)

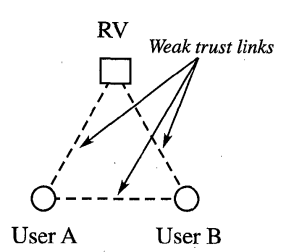

(b)

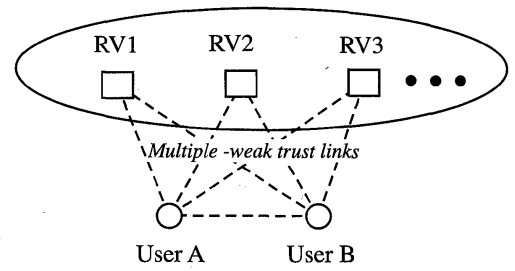

(c)

Fig. 5 Comparison of moderate trust binding of our scheme with the PKI trust model. (a) In PKI, trust between two peers is derived from the two strong trust bindings between $\mathrm{CA}$ and each peer. (b) Our scheme generates three moderate trust bindings between three tiers <initiator - delegate target $\rangle$. (c) Strengthening trust binding by repeating our scheme several times using multiple delegates. 
between users and the CA is a strong one, and the trust between users is a derivation of the strong trust. This is illustrated in Fig. 5 (a).

On the other hand, our scheme uses an instantly generated moderate trust link through a randomly selected delegate (as shown in Fig. 5(b)) or through multiple random delegates (as shown in Fig. 5 (c)). If the delegate(s) are selected randomly enough, it will make it very hard for an attacker to predict the exact identifier of the delegate (blinding attacker). By combining the identifier of a peer into the moderate trust link, an attacker cannot do much harm (such as misrouting, corrupting, or dropping communication data) to the initiator or the target (moderate trust). Therefore, if an attacker tries to launch any identifier related attack, it must break the moderate trust binding(s).

To combine the identifier of peers into the moderate trust binding, our approach is also motivated by two mechanisms called an ownership proof scheme and an exclusiveness proof scheme.

\subsubsection{Ownership Proof Scheme}

The public key cryptography-based ownership proof that we consider in this paper was originally proposed in [16] and subsequently adopted in many applications [17], [18]. In a distributed environment, the scheme presents a simple but strong enough mechanism to prove ownership of identity without requiring any central agency or infrastructure such as PKI.

The underlying concept is that when a public key is used as an input to the identifier generation function $f$, such that

$$
i d=f(\text { public key })
$$

then the $i d$ can be proved to be owned by the private key holder using

$$
v(\text { id, public key) } \rightarrow \text { \{success, fail }\} .
$$

A commonly accepted identifier generation function in P2P networks is using a cryptographic hash like MD5 or SHA1. However, we should note that the ownership proof itself is very weak to be used for identifier authentication. Therefore, it must be used in conjunction with other schemes.

\subsubsection{Exclusiveness Proof Scheme}

In a network, exclusiveness means that an identifier id cannot be used in the same network for another node. If there is any such scheme, then it will provide more powerful security than the ownership proof does. To our knowledge, however, no scheme is known to achieve exclusiveness in distributed P2P networks.

Alternatively, recent researches provide proof of moderate exclusiveness by including IP address information in the input of identifier generation function $f$. If an identifier is generated by

$$
i d=f(i p), \quad i p \text { is an IP address, }
$$

then interim routing nodes or any external attacker cannot spoof the identity. More discussion about binding an IP to an identifier can be found in several literatures [10].

\subsection{Random Visitor}

Let $\mathcal{P}$ be a set of nodes participating in a peer-to-peer (P2P) network. Let $\mathcal{D} \subset \mathcal{P}$ be another set of nodes participating in a peer-to-peer (P2P) network (The relation between the $\mathcal{D}$ and the $\mathcal{P}$ will be further extended in later section.). We consider a situation in which an entity tries to verify the identity of another peer, where the former we call an initiating peer (or simply an initiator) and the later we call a target peer (or simply a target). A P2P network is fully dynamic in time and space, allowing changes of location and identifier. Then, a random visitor is defined as follows:

Definition 1 (Random Visitor). A random visitor is a randomly selected network node $d \in \mathcal{D}$ that is serving as a delegate of an identity proof procedure triggered by an initiator $i \in \mathcal{P}$ against a target $t \in \mathcal{P}$. The random visitor needs not be trusted by the initiator or the target, or vice versa; nor have any shared secret with the initiator or the target. The identity proof is unidirectional.

The random visitor in our approach is a delegate that actually carries a key material $\epsilon$ (to be defined later) that can be used by the target to prove his own identity to the initiator. As the name implies, the delegate is selected randomly from a delegate space $\mathcal{D}$. Furthermore, because any randomly selected third party can be a delegate, there is no pre-configured credential between the random visitor and the initiator (or the target). In the later subsection, we will discuss various classes of $\mathcal{D}$; for now just treat it as a group of network nodes.

When evoked (or visited) by a random delegate, the target should respond within due time, $t<t_{s}$, where $t_{s}$ is the sojourn time of the delegate. If the target either fails to manipulate $\epsilon$ and prepare a response, or fails to respond to the initiator within $t_{s}$, then the initiator judges that the identity of the target has been compromised (i.e., there is an on-going Sybil or Eclipse attack).

For easy reference, we will refer to the random visitor scheme as $F_{R V}$. It is a function defined as

$$
F_{R V}: \mathcal{P} \times \mathcal{I} \times \mathcal{T} \rightarrow\{\text { success, fail }\}
$$

That is, $F_{R V}$ maps an ordered pair (unidirectional) of peers to a value of success or fail at the evaluation time. If $F_{R V}\left(p_{i}, i d_{k}, t\right)=$ success, then the identity of target $i d_{k}$ is proved to be authentic in the perspective of initiator $p_{i}$, at time $t\left(t_{0} \leq t \leq t_{0}+t_{s}\right)$. $t_{0}$ is the time at which the initiator has requested service of the random delegate.

Because an identity attack such as Sybil or Eclipse is closely related to the routing path from the initiator to the 
target, $F_{R V}\left(p_{i}, i d_{k}, t\right) \neq F_{R V}\left(p_{j}, i d_{k}, t\right)$ when $p_{i} \neq p_{j}$. Furthermore, because the P2P network is dynamic, it is not always guaranteed that $F_{R V}\left(p_{i}, i d_{k}, t_{1}\right)=F_{R V}\left(p_{i}, i d_{k}, t_{2}\right)$ when $t_{1} \neq t_{2}$. If the identity of the target changes, i.e., from $i d_{k}$ to $i d_{k}^{\prime}\left(i d_{k} \neq i d_{k}^{\prime}\right)$, then $F_{R V}\left(p_{i}, i d_{k}, t_{1}\right) \neq F_{R V}\left(p_{i}, i d_{k}^{\prime}, t_{2}\right)$, where $t_{2}=t_{1}+\delta(\delta>0)$.

Our scheme $F_{R V}$ achieves moderate exclusiveness by introducing identity-based cryptography for the random visitor scheme such that "only one who is bound to an initiator through a randomly selected delegate can respond to the initiator successfully using the information stored in the key material $\epsilon$." As a result, $F_{R V}$ makes the attacker blind or partly sighted (who can only see the initiator and the target). Furthermore, the location of the target is also tied to the $\epsilon$ so that the scheme achieves network-bound exclusiveness. The chance that an attacker can compromise the scheme is also limited by the sojourn time $t_{s}$.

\subsection{On-Line Registration Metaphor}

A simple metaphor that is commonly found in everyday Internet service can be used to present the underlying concept of the random visitor.

As an example, consider that Bob is trying to register himself to a Web server through the Internet. The scheme, which we call "registration by e-mail confirmation," states that "to confirm your registration, we will send an e-mail to the e-mail address you have submitted. You must reply to the e-mail within 12 hours to complete your registration. If you do not, the registration information will be deleted from the file."

In this example, Bob is a target whose identity needs to be proved, and the web server who sends the confirmation message is an initiator. The e-mail server acts as a delegate. The overall procedure is presented in Algorithm 1. During user registration to a web-based service, identity of a user can be secured by a trust binding provided by a email server. Assume that a use Bob has an e-mail account bob@abc.com. After this algorithm has been performed, the web server can verify whether Bob's identity is valid or not within the domain abc.com (Lemma 1). If the algorithm is performed for another e-mail account bob@def.com, then it assures that the identity is also valid within def.com (Lemma 2).

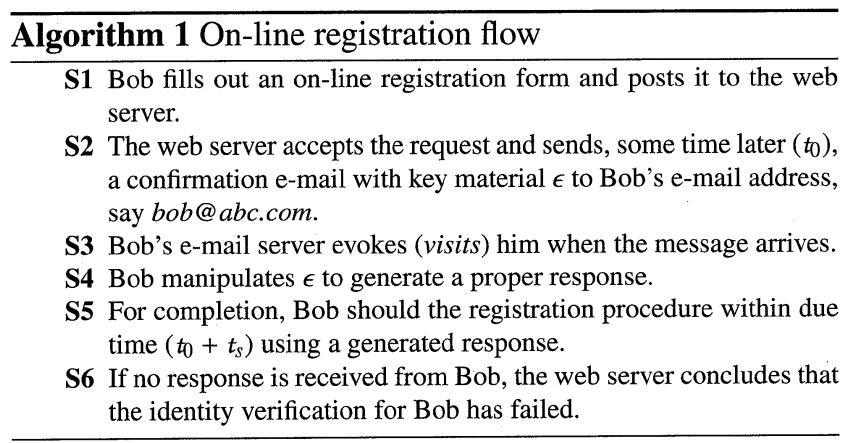

Though the delegate, the e-mail server, is a static one, this simple example captures a number of important characteristics of the random visitor, each of which is described separately below.

- Moderate trust on the user: Trust of the above procedure depends (1) primarily on the fact that the key material $\epsilon$ can only be accessible by the one who has access to the e-mail address, namely bob@abc.com, and (2) partly on the fact that Bob is already a registered user of the e-mail server. If Bob cannot access the e-mail or fails to respond within due time, the online registration fails.

- Deduced trust: If the web server has little or no trust for the e-mail server, so it does for Bob. Therefore, the trust is deduced from the chance by which the email server will visit Bob and deliver $\epsilon$ successfully. The 'trust' in this case means that the e-mail server is not being attacked with high probability, and it does not mean that the e-mail server and the web server have any pre-configured credential (i.e., shared secrets or public key certificates issued by a mutually trusted third party).

- Empirical tight binding: Several aspects of the registration procedure create tight bindings between the web server and the e-mail server, and between the email server and Bob such that they are enough to prevent possible attacks. That is, if an attacker is trying to forge the registration procedure from Bob's side, he must crack the binding between Bob and the e-mail server; if he/she is trying to attack from the side of the web server, he/she must crack the binding between the web server and the e-mail server. Breaking the communication path from Bob to the web server is not enough. In the example, it is also not defined when and how the web server will solicit a proxy from the e-mail server, which decrease or limit the possibility an attacker can try some predefined attack.

\section{The Framework}

In this section, we present an overall description of our framework and several constituents. We start the discussion by introducing a noble public key cryptography, called identity-based cryptography.

\subsection{Identity-Based Cryptography}

In this paper, we use the idea of identity-based cryptography (IBC) [12]-[15], [37] to fulfill the design principles of a P2P overlay network proposed in [31] so that we can defend against identifier-related attacks such as Sybil [11] and Eclipse [29].

Public-key cryptography was designed to solve the online key distribution problems of symmetric key cryptography [38]. But it also has a drawback; that is, the identity of the public key holder should be bound to the public 
key of that holder. This is called a key authentication problem. The Public Key Infrastructure (PKI) is a collection of technical and organisational facilities that provides a trusted third party to attest to the key authentication problem. The trustworthy third party is arranged to publish trust materials, called certificates, which can be used to validate the binding between a user and his or her public key. Therefore, to send a secure message to someone else encrypted with his public key, a certificate for that public key should be acquired and verified.

The concept of identity-based cryptography was originally introduced by Adi Shamir in 1984 [12] and has been studied in the cryptography context. The purpose of the IBC is to eliminate the demanding task of collecting public key certificates of peers and verifying the signatures signed by some trusted third party. It simply uses well-known, selfevident identity information as the public key of a peer. Any globally unique identity information can be used for this purpose, including an e-mail address, a phone number, or an IP address. The original cryptographic scheme proposed by Shamir [12] was a signature scheme. Recently, two identitybased encryption schemes were proposed in [13], [14] and were proved to be secure and practical [15]. See [15] for a more general discussion.

When Alice wants to send a message to Bob, for example, she signs it with her private key, encrypts the result using the recipient's name, "Bob", appends her own name, "Alice", and sends the result to Bob. Then Bob decrypts the message using his own private key and verifies the signature using the sender's name, "Alice". In fact, the private keys owned by Alice and Bob are created by a trusted third party, called a private key generator (PKG), using their respective names, "Bob" and "Alice" [12]. The private key is generated by the PKG using [39, pp. 434-457]

private key $=F_{p r i}($ master secret, public identity $)$,

where the master secret is only known by the PKG. The PKG sends the resulting private key to the holder of the public identity through a secure channel possibly at the bootstrapping time. Shamir's suggestion for the secure channel uses an off-line smart card distribution. Along with a private key, the PKG also sends several system parameters that are common to all users. The content of the parameters differ from IBC to IBC. A generic structure for identity-based encryption is shown in Fig 6.

Note that the names such as "Bob" and "Alice" are publicly known values, so there are some parameters in the IBC mechanisms that are known only to a single user to ensure that only the correspondent can decrypt a cipher message or generate a signature.

\subsubsection{Applying IBC to the Random Visitor Scheme}

Without providing details of the respective method, this paper treats the identity-based cryptographic (IBC) scheme as a "black box" to embed an identifier into the trust binding. Thus we assume that there are two IBC's, $E_{i d_{k}}\left(D_{i d_{k}}\right)$

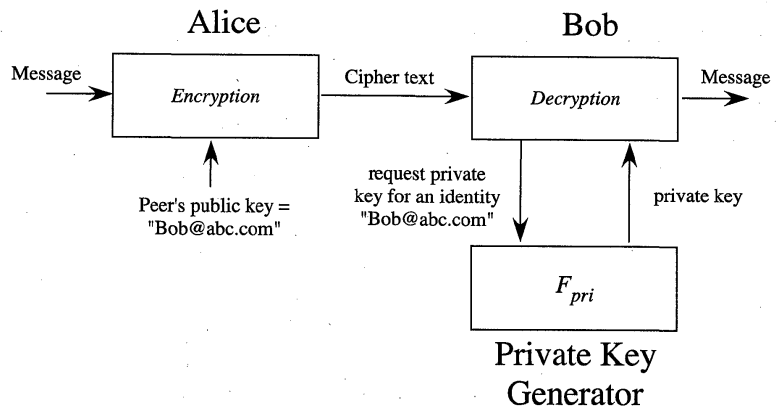

Fig. 6 Overall operation flow of an identity-based encryption system.

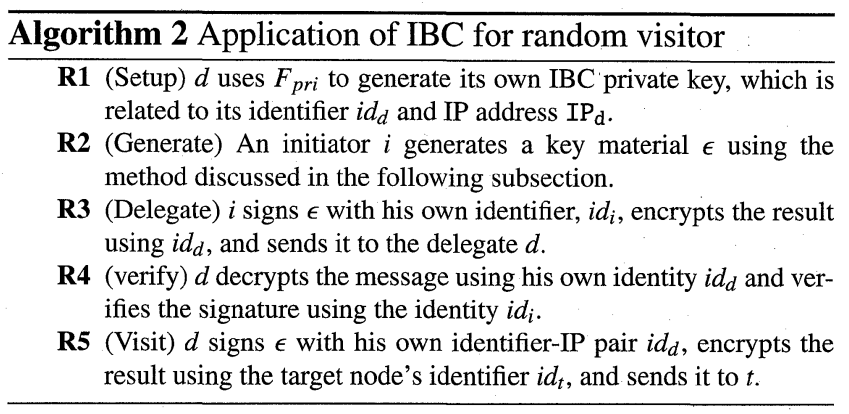

and $S_{i d_{k}}$, for en(de)cryption and signature, respectively. The IBC functions used in this paper are defined as

$$
\begin{aligned}
& \text { cipher text }=E_{i d_{k}}(\text { plain text }), \\
& \text { plain text }=D_{i d_{k}}(\text { cipher }), \\
& \text { signature }=S_{i d_{k}}(\text { plain text }),
\end{aligned}
$$

where $i d_{k}$ is the public identity of a node $k$.

Although IBC is simple and efficient, it has a drawback in that the private key generator $(P K G)$ is required to generate the private key of the recipient of the message. Our scheme eliminates the requirement by making each peer generate its own private key. That is, the $F_{p r i}$ is performed by the public identity holder. On the surface, this approach may seem to have the same key authentication problem of the PKI, but the insurance it provides is quite inexpensive, and sufficiently tight binding between the sender and the receiver of a message is possible. To be able to attack the verification mechanism, an attacker should break the binding. Besides, we should note that we are not dealing with the key authentication problem, but the hardening identifier security ${ }^{\dagger}$.

Algorithm 2 provides overall scenario in which the IBC is applied to the random visitor scheme. In the algorithm, an

${ }^{\dagger}$ To relax the PKG-related issue and to provide much strength to the overall procedure, the random visitor scheme may additionally use a proxy-based $I B C$ as suggested by Mambo, et al. [40]. Instead of generating private keys of each peer, the PKG can issue identifiable proxy of his/her signature. A peer appends proxy signature in its IBC operations. Because the proxy is identifiable (who has appended the proxy signature) and verifiable (whether the proxy signature is valid), other peers can verify that the IBC encryption or signature is made by the peer. 
initiator $i$ wants to verify identity of a remote peer $t$ by soliciting a proxy peer $d$. It is assumed that each peer $i, t$, and $d$ has publicly known identifier $i d_{i}, i d_{t}$, and $i d_{d}$, respectively. After this algorithm has been performed, the peer $i$ can verify whether the identity of the remote peer $t$ is valid or not within the context of the proxy peer $d$ (Lemma 1). If the algorithm is performed for another proxy peer $d^{\prime}$, then it assures that the identity is also valid within the context of the second proxy peer $d^{\prime}$ (Lemma 2).

The Algorithm 2 only includes partial operations of the random visitor scheme. In general, multiple delegates are solicited in tandem or in parallel during the protocol. Thus the operations $\mathbf{R 3}$ - R4 (or R1 - R5) will be iterated multiple times between such delegates. We will complete the procedure in Sect. 4.3 when we introduce a two phase random visitor scheme.

\subsection{Key Material $\epsilon$}

In stating the concept of the random visitor scheme, we have left the details of the key material $\epsilon$ unaddressed. In summary, together with identity $i d$, the key material $\epsilon$ is used to evaluate the ownership of an identifier.

A recently developed method to prove ownership of an identity in a distributed environment is to generate the identity from the public key of the owner by using a mechanism similar to CAM [16]-[18]. For this purpose, the identifier of a node $k$ is generated as

$$
i d_{k}=H\left(K_{k}^{u} \mid \mathrm{IP}_{\mathrm{k}}\right),
$$

where $K_{k}^{u}$ is the RSA public key and $\mathrm{IP}_{\mathrm{k}}$ is is IP address of a node $k$. In this paper, we will use a modified version of this format as given in equation (5) so that the proposed scheme can verify the bindings between three tuples: ID, IP address, and self-generated public/private key pair.

When an initiator $i$ tries to verify target identifier $i d_{k}$ through a delegate $d$, the key material $\epsilon$ is generated by the initiator as follows:

$$
\epsilon=E_{i d_{k}}\left(N_{v} \mid N_{k}\right),
$$

where $N_{v}$ and $N_{k}$ are nonces generated by the initiator and the target, respectively. In other words, the $\epsilon$ is an encrypted value of the two nonces using the target identifier $i d_{k}$ as the key. Therefore, secrecy of the IBC guarantees that only the target can see the nonce $N_{v}$.

The use of $i d$ and $\epsilon$ achieves that "the one who can properly manipulate the key material $\epsilon$ owns the auxiliary identifier id, thus the ID is tied to that node."

\subsection{Protocol Details}

In a dynamic overlay network, if a node $i$ tries to connect to a target node $t$, it should verify that the identifier of the node $t$ is authentic, i.e., created and owned by the claimer. We propose an identity verification mechanism, called a random visitor scheme, which works in two phases (see Fig. 7).

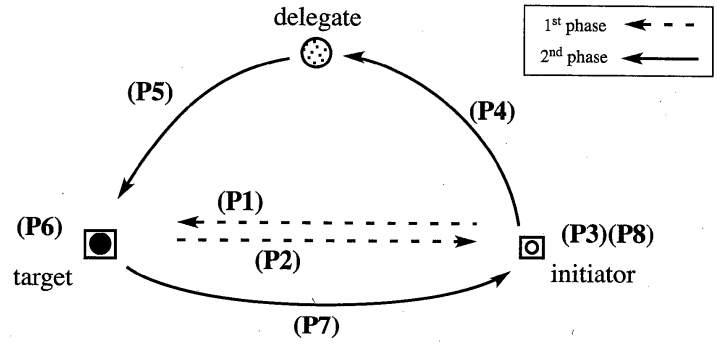

Fig. 7 Overall random visitor procedure. The labels along the lines correspond to the protocol steps in the Algorithm 3.

We assume that the network is dynamic so that the identifier of each peer or their location (IP address) changes. However, whenever such change is made, the peer who made such change is responsible to perform an identifier management function $F_{A}$ to keep the overlay synchronized [41], [42]. We also assume that each node has a fixed, globally unique user identifier ID. Therefore, using our identifier proof mechanism means to prove that the ID is strongly tied to the IP address of the node in such a way that the ownership is verifiable and the ID-IP pair is used exclusively in the P2P network. These are achieved by using an auxiliary identifier aid. For a node $t$, its auxiliary identifier $a i d_{t}$ is created by using a hash function:

$$
\operatorname{aid}_{t}=H\left(\mathrm{ID}_{\mathrm{t}}\left|K_{t}^{u}\right| N_{i}\left|N_{t}\right| \mathrm{IP}_{\mathrm{t}}\right),
$$

where $\mathrm{ID}_{\mathrm{t}}$ is the globally unique identifier of $t, K_{t}^{u}$ is the public key generated by $t$ during the bootstrapping procedure, $N_{i}$ is a nonce included in an initial request message sent by the initiator, $N_{t}$ is a nonce generated by $t$, and finally $\mathrm{IP}_{\mathrm{t}}$ is the IP address of $t$.

When an initiator $i$ tries to verify target identifier $\mathrm{ID}_{\mathrm{t}^{\prime}}$ $\mathrm{IP}_{\mathrm{t}}$, it verifies $\operatorname{aid}_{t}$ through a delegate $d$. Therefore, a new $\operatorname{aid}_{t}$ is generated whenever an identifier verification is required between two nodes.

The overall $F_{R V}$ protocol is shown in Algorithm 3. In this algorithm, an initiator $i$ wants to verify identity of a remote peer $t$ by soliciting a proxy peer $d$. It is assumed that each peer $i, t$, and $d$ has publicly known identifier $\mathrm{ID}_{\mathrm{i}}, \mathrm{ID}_{\mathrm{t}}$, and $I D_{d}$, respectively. After this algorithm has been performed, the peer $i$ can verify whether the identity of the remote peer $t$ is valid or not within the context of the proxy peer $d$ (Lemma 1).

Before the identifier verification protocol is started (P0), it is assumed that the initiator has acquired the peer's identifier information through some mechanism such as an advertisement or explicit search using $F_{R}$. For example, the initiator may find the IP address of the target using an ordinary lookup mechanism, such as Pastry [23]. If the network is dynamic, the IP address of a peer can only be found by using $F_{R}$, assuming that the peer is on-line and has performed an identifier allocation using $F_{A}^{\dagger}$. Basically, we are assuming an application scenario in which the initiator collects

${ }^{\dagger}$ The $F_{R}$ can be any discovery mechanism of DHT-based overlay network or P2P framework such as JXTA [26]. 


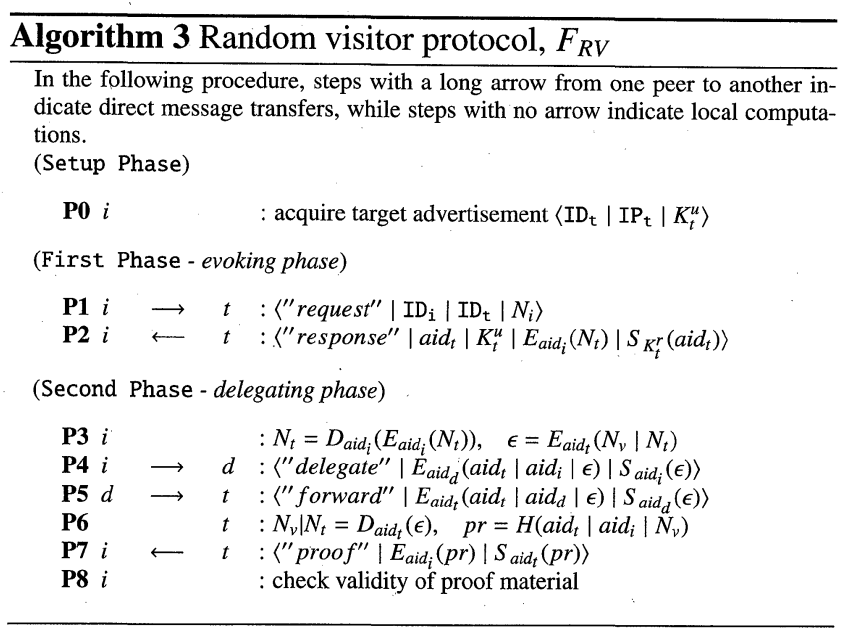

a list of peer nodes in the P2P network at the time of the bootstrapping of the initiator. Thus, after the initiator has acquired the IP address of the target, it needs to verify the authenticity of the ID - IP pair.

In the first phase $(\boldsymbol{P 1}-\boldsymbol{P 2})$, the initiator sends a request for an identifier verification protocol to the target (P1). The message contains the identifier of the initiator and the target. The nonce $N_{i}$ in the request message ensures that the identifier verification is fresh. Upon receiving the request message, the target generates a new auxiliary identifier aid $_{t}$ using equation (5) and sends a response message back to the initiator (P2). The nonce $N_{t}$ generated by the target is used as a secret for identifier verification. Thus to make the $N_{t}$ be available only to the initiator, it is encrypted using the initiator's public auxiliary identifier, $\left(a i d_{i}\right)$. The IBC ensures that only the one who owns aid $_{i}$ and its related IBC parameters can decrypte $E_{a i d_{i}}\left(N_{t}\right)$.

As an additional feature, the procedure $\mathbf{P 2}$ provides ownership of identifier $\mathrm{ID}_{\mathrm{t}}$ and the RSA private key $K_{t}^{r}$ by combining a public key signature (i.e., $S_{K_{t}^{r}}\left(\right.$ aid $\left._{t}\right)$ ) into the message (c.f., Sec. 3.1.1), which can be used immediately or later for session key establishment ${ }^{\dagger}$.

In the second phase (P3 - P7), the initiator generates a key material $\epsilon$ using the response from the target (P3), and delegates the proof mechanism to a randomly selected node $d \in \mathcal{D}(\mathbf{P 4})$. The key material is generated using the equation (4). The selection of a random delegate will be discussed shortly below. The delegation message is signed using $S_{s i d_{i}}(\epsilon)$ so that the delegate $d$ can verify the authenticity of the message and thus binding $\mathrm{ID}_{\mathrm{i}}-\mathrm{IP}_{\mathrm{i}}$. On receiving the delegation message, the delegate simply forwards $\epsilon$ to the target. To do this, the delegate reproduces its own delegate message and appends its own signature (P5).

When the target $t$ receives $\epsilon$ from the delegate, it can decrypt it and prepare a proof material $p r$ (P6). The target sends $p r$ along with its signature to the initiator (P7). The initiator checks the signature and the hashed proof $p r$. If the hash matches, the identity verification is completed $(\mathbf{P 8})$ with a result success.

In the steps P4 and P5, part of the messages are pro- tected by using encryption functions $E_{a i d_{d}}$ and $E_{a i d_{t}}$, respectively.

The proposed random visitor scheme provides ownership proof and moderate exclusiveness for a given identifier ID, which is claimed to be held by an entity who is using a given IP address. Without the proof, an attacker can launch a Sybil or Eclipse attack by placing a forged identifier near enough, in the identifier space $\mathcal{I}$, enough to the target or to the initiator.

Because the $a i d_{t}$ is generated using a hash function which takes the target's RSA public key and IP address as its input, it provides ownership proof of public key $K_{t}^{u}$ that cannot be spoofed by any other node: Furthermore, as the key material $\epsilon$ is conveyed by a randomly selected delegate $d$, an attacker has not enough time to place a forged identifier near the random visitor. In other words, if an attacker tries to launch a Sybil or Eclipse attack, he must forge large enough identifiers so that any randomly selected $d$ resides within the range of the forged identifiers with a very high probability, which limits the possibility of a successful identity attack.

As the identifiers and the IP addresses of the initiator, delegate, and the target are included in the delegation messages, they also provide moderate network-bounded exclusiveness.

\subsection{Expanding' the Random Visitor Scheme}

As discussed in the previous subsection, the strength of the random visitor scheme partly relies on the random selection of the delegate $d$ from the delegate space $\mathcal{D}$ such that an attacker faces the difficulties of placing some forged identifiers near the randomly selected delegate in due time before the protocol starts and of preparing proper a response before the expiration of $t_{s}$.

To decrease the possibility of a successful attack, we can make a slight modification to the second phase to introduce multiple tandem or parallel delegates, thereby increasing the difficulty of identity forgery. Two different realizations of multiple delegates are shown in Fig. 8. When the initiator employs $\ell$ different delegates in tandem, as sown in Fig. 8 (a), the steps (P4-P5) are expanded $\ell$ times to be

$$
i \rightarrow \underbrace{d_{1} \longrightarrow d_{2} \longrightarrow \cdots \longrightarrow d_{\ell}}_{\ell \text { delegates in tandem }} \rightarrow t
$$

where right arrow " $\longrightarrow$ " indicates message transfer from one peer to another.

Because this is a series system, the possibility of a successful identifier proof mechanism may decrease quickly with an increase in $\ell$ due to the product law of probability [43, pp. 25-36]. For example, if the communications in a P2P network has reliability of 0.97 and $\ell=5$, then the possibility of successful identifier verification decreases to 0.858 .

If the initiator utilizes $\ell$ delegates in parallel, as shown

${ }^{\dagger}$ Note that the protocol works even if the public key signature $S_{K_{t}^{r}}\left(\right.$ aid $\left._{t}\right)$ is not combined. 


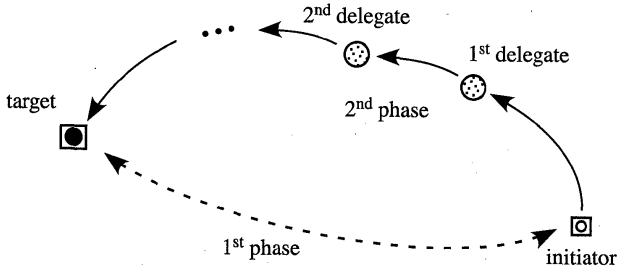

(a)

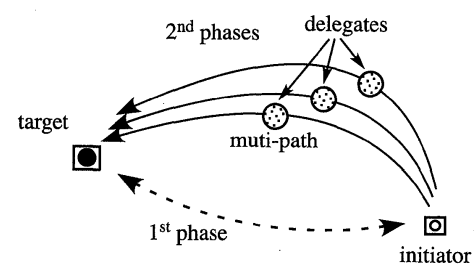

(b)

Fig. 8 Expansion of random visitor scheme: (a) tandem delegates and (b) parallel delegates

in Fig. 8 (b), the steps (P3-P8) are repeated $\ell$ times. If there are $\ell$ delegates, they harden the identifier security $\ell$ times more than a single delegate case. The strength of this approach and the appropriate value of $\ell$ will be presented in Sect. 5 .

We still need to clarify the selection mechanism for multiple delegates, which will be presented when we discuss several classes of $\mathcal{D}$ in the next subsection.

\subsection{Special Classes of the Random Visitor}

In Sect. 3.2, the random visitor space $\mathcal{D}$ is defined as a subset of the $\mathcal{P}$, set of nodes participating in a peer-to-peer (P2P) network. In this section, we will extend the relation. That is, we consider a random visitor with special structures by classifying the delegate space $\mathcal{D}$.

Central: This is a special class of random visitor where $|\mathcal{D}|=1$ and $\mathcal{D} \not \subset \mathcal{P}$. That is, the delegate is not random but rather a static one. A diagram shown in Fig. 9 illustrates this class. If the delegate is not random, it seems unlikely that the possibility that an attacker can launch an identity attack decreases by introducing our two-phase random visitor scheme. However, managing such a fixed delegate acts as a strong invariant to defend against attacks such Eclipse (c.f., $P 1$ and Lemma 2). When a peer is completely isolated from the network as a result of an Eclipse attack, then it cannot proxy the fixed delegate. Once a peer can reach the fixed delegate, on the other hand, it can start identifier verification from that point. If we increase $|\mathcal{D}|$, the strength of the network against the Eclipse attack will also increase.

Integrated: In this class, $\mathcal{D}$ is constructed from the list of peers found from the overlay network itself by using $F_{R}$ or other discovery mechanism. Therefore, $\mathcal{D} \subset \mathcal{P}$. If the scheme is performed in a DHT-based overlay network, the first and second phases of the random visitor scheme will look like using two different routing paths such that

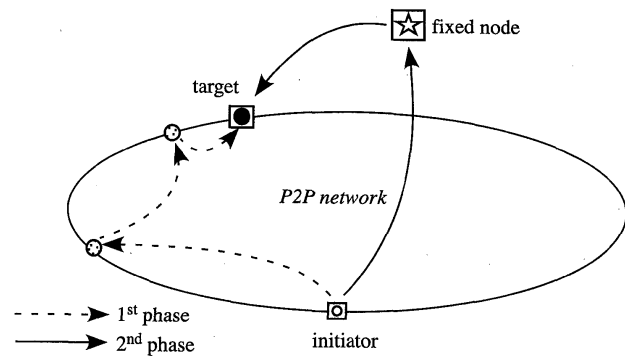

Fig.9 A centralized random visitor configuration.

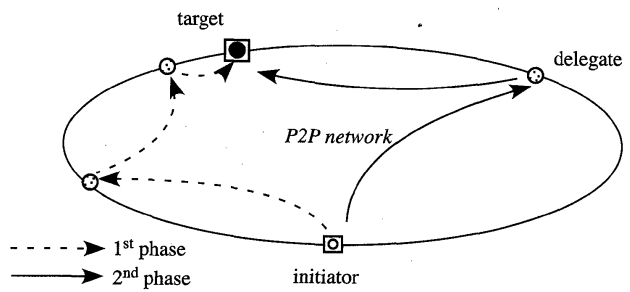

(a)

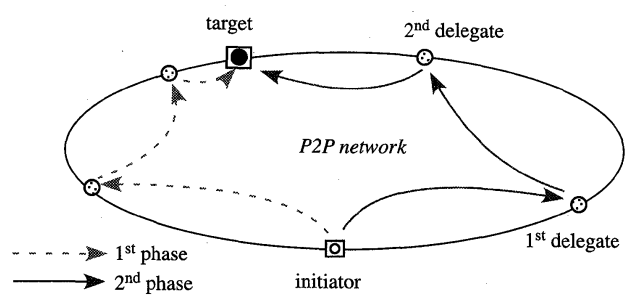

(b)

Fig. 10 An integrated random visitor configuration: (a) single delegate (b) multiple delegates in tandem.

$\mathrm{IP}_{\mathrm{t}}=F_{R}\left(\mathrm{ID}_{\mathrm{t}}\right)=F_{R}\left(\right.$ aid $\left._{t}\right)$. This is illustrated in Fig. 10 . Because the two identifiers $\mathrm{ID}_{\mathrm{t}}$ and aid $_{t}$ are not equal to each other, the result has a different routing path and length. (Note that the number of the hops of the first and the second phase message delivery may differ from implementation to implementation.)

Split: This class is similar to the integrated one, but differs in the fact that the second phase is separated from the first phase. The second phase is an isolated one, possibly another overlay network that is different from the one in which the initiator and the target reside. We can call the the former a second ring and the latter a first ring. When the request message is received, the initiator and target become a cloned one of the second ring. Thereby the RV scheme starts from the cloned initiator and ends at the cloned target. This is illustrated in Fig. 11. Through this configuration, peers in a separate $\mathrm{P} 2 \mathrm{P}$ network can act as service providers, which are dedicated for identifier verification of two network peers (regardless of whether they are members of a P2P network or not). 


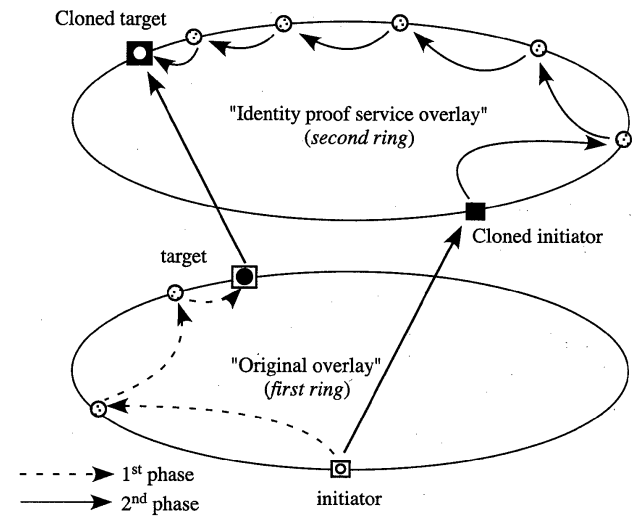

Fig. 11 A split random visitor configuration. Peers in a separate P2P network can act as service providers, which are dedicated for identifier verification of two network peers (regardless of whether they are members of a P2P network or not).

\subsubsection{Random Visitor as a Service}

The split class of a random visitor can be further generalized to be used for an identity proof service (IPS) model. In other words, any node that belongs to a global P2P overlay network and who wants to verify identity of another peer sends a request to the peer. Then the two parties can clone themselves by generating $\operatorname{sid}_{t}$ and $s i d_{i}$; and join to the IPS overlay (the second ring) to perform the second phase of the random visitor scheme. This service model suggests that peers in an overlay network can collectively become a service-provisioning entity to another overlay network.

\section{Security Analysis}

In the first and second phases of the random visitor scheme, messages between three tiers $\langle$ initiator - delegate - target $\rangle$ are secured using a moderately strong and computationally efficient mechanism based on identity-based cryptography (IBC). IBC is a relatively new technology, and a lot of new results have been achieved in recent years. Security of the IBC related operations in the random visitor is similar to that of the $\mathrm{IBC}^{\dagger}$, which is generally accepted to be secure and practical.

Without the help of a trusted third party as a private key generator $(\mathrm{PKG})$, there is the same key authentication problem because the $F_{p r i}$ is performed by the public identity holder (c.f., Sect.4.1). This is the reason why we say the scheme has "moderate security." However, we should note that the strength of the random visitor scheme does not rely on the authenticity of the IBC key held by each peer node. More precisely, the proposed scheme depends on the basis that the collection of such moderately secure bindings is enough to make it difficult for an attacker to perform an identifier forgery attack. As discussed in Sect. 4.1, the proxy signature scheme [40] can improve the security of this approach.

If an attacker has attacked a sufficiently large set of

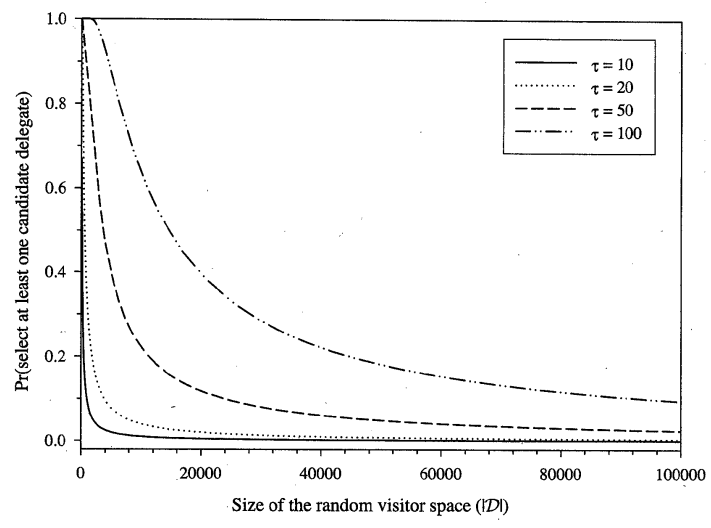

Fig. 12 Probability of selecting at least one delegate that has already compromised assuming that an attacker can compromise $\tau$ nodes from the total delegate space $|\mathcal{D}|$.

identifiers that belong to $\mathcal{D}$, there is the possibility that the initiator may select, as a random visitor, a node that has already been compromised by the attacker. We refer to this situation as a collision. In this case, the attacker can easily control the binding between the initiator and the delegate, or the binding between the delegate and the target. Even in such a situation, however, the attacker cannot cause any other malicious harm but to deny being a proxy of the identifier verification procedure and to ignore the delegation request message. The initiator will eventually time out and try another delegate.

Assuming that an attacker can compromise randomly selected $\tau$ nodes from the delegate space $\mathcal{D}$, and assuming that the initiator can only see another random candidate list of $\tau$ nodes from the same delegate space, a rough estimation of the collision probability $P_{c}$ that at least one of the attacker's selection belongs to the initiator's candidate list is

$$
P_{c}=\operatorname{Pr}(\text { Select at least one candidate list }) \text {. }
$$

We found that a technique [44], [45] for analyzing the solution for key predistribution in sensor networks can also be applied, with a little modification, to find $P_{c}$ as

$$
\begin{aligned}
P_{c} & =1-\frac{\left(\begin{array}{l}
|\mathcal{D}| \\
\tau
\end{array}\right)\left(\left.\right|_{\tau} ^{|\mathcal{D}| \tau)}\right)}{\left(\left.\right|_{\tau} ^{|\mathcal{D}|}\right)^{2}} \\
& =1-\frac{((|\mathcal{D}|-\tau) !)^{2}}{(|\mathcal{D}|-\tau) !(|\mathcal{D}|) !} .
\end{aligned}
$$

Figure 12 shows several plots of this function. The figure indicates that for a network with $|\mathcal{D}|=100,000$ delegates, even when an attacker can compromise 100 nodes, the probability of a successful attack is 0.09 . This points out that randomly selecting a delegate from a space still has low collision probability if the space is large enough. Figure 13 shows the desired size of the random visitor space $|\mathcal{D}|$ to

${ }^{\dagger}$ Security of the IBC proposed by Shamir [12] is similar to that of the RSA. That is, it depends on two mathematically difficult problems: 1) the problem of factoring large numbers; and 2) finding $e^{\text {th }}$ root modulo $n$ when $n$ is a composite number whose factors are not known. 


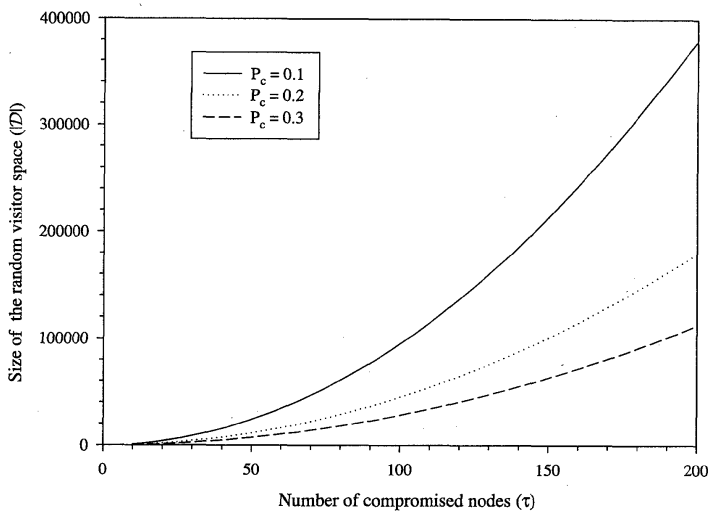

Fig. 13 Desired size of the random visitor space when an attacker can compromise $\tau$ nodes.

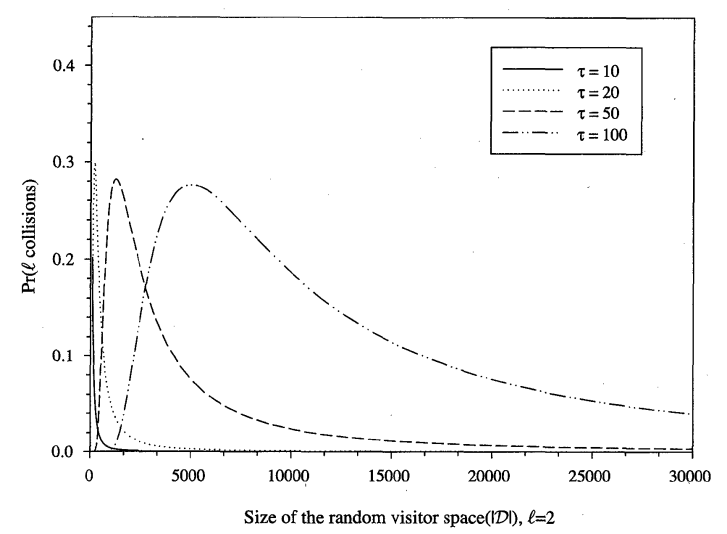

(a)

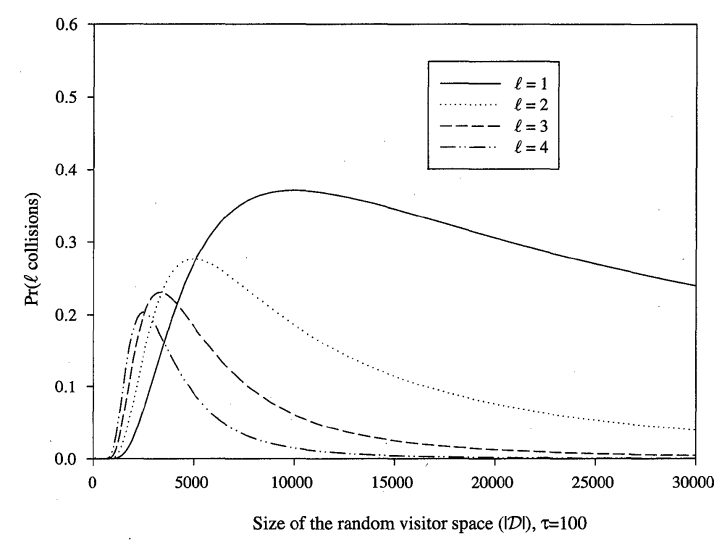

(b)

Fig. 14 Probability of $\ell$ collisions (a) for various values of $\tau$ when $\ell=2$, (b) for various values of $\ell$ when $\tau=100$

keep the probability of collision $P_{c}$ low enough (around 0.1 , 0.2 , and 0.3 , respectively).

In order to obtain the desired possibility of successful identifier verification, the random visitor scheme should be performed multiple times. Let $\ell$ denote the number of successive trials and $p(i)$ be the probability that there is exactly $i$ successive collisions. That is,

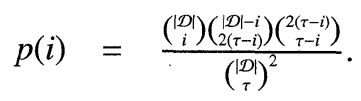

Note that the $P_{c}$ is a special case of $1-p(0)$. When an initiator performs $\ell$ verifications, the attacker can succeed only when it has forged all $\ell$ delegates. Thus the probability of $\ell$ collision is

$$
\begin{aligned}
P_{c(\ell)} & =p(\ell) \\
& =\frac{\left(\begin{array}{l}
|\mathcal{D}| \\
\ell
\end{array}\right)\left(\begin{array}{c}
|\mathcal{D}|-\ell \\
2(\tau-\ell
\end{array}\right)\left(\begin{array}{c}
(\tau-\ell) \\
\tau-\ell
\end{array}\right)}{\left(\begin{array}{l}
|\mathcal{D}| \\
\tau
\end{array}\right)^{2}} .
\end{aligned}
$$

An evaluation of equation (9) is shown in Fig. 14. In Fig. 14 (a), we compared the probability of $\ell$-collisions $P_{c(\ell)}$ for a fixed $\ell=2$ while $\tau$ is set to $10,20,50$, and 100. Figure 14 (b) compares $P_{c(\ell)}$ for a fixed $\tau=100$ while $\ell$ varies between 1 and 4 . Compared to the Fig. 12, these diagrams show that by repeating the random visitor scheme the network is still resilient for 100 identifier forgeries from relatively small delegate space $\mathcal{D}$. When $|\mathcal{D}| \approx 10000, P_{c(\ell)}$ is less than 0.02 even if an attacker can forge more than 100 identifiers. Figure 14 (b) also indicates that the optimal value for $\ell$ is 3 or 4 depending on the size of the network.

Because our analysis is based on some assumptions about $\mathcal{D}$ and $\tau$, the result in Fig. 14, when the network size is less than 6000, seems undesirable. As discussed in Sect. 4.5, fixed delegate(s) can be introduced in such networks so that they can act as a strong security anchor point.

\section{Implementation and Measurements}

We have designed and constructed a prototype testbed of peers, which implement a random visitor protocol for the identifier verification in Section 4.3. The experiments are carried out on three Pentium-IV machines running Windows XP. The machines are connected by a $100-\mathrm{Mbps}$ Ethernet. Cryptographic routines from Crypto++ library are used for Triple DES encryption and RSA signature [46]. The IBC routines are implemented using the GNU Multiple Precision Arithmetic (GMP) library [47].

In our implementation, the signature is based on the original proposal of Shamir [12], and the encryption is realized by the standard DES with key agreement based on Okamoto [37]. For higher performance, we may use other IBC schemes like [14], which uses elliptic curve pairing and achieves high speed encryption and decryption compared to the integer factoring schemes [12], [37]. For the implementation of a public key signature in P2 and P3, a 1024-bit RSA signature and verification are used.

For the experiment, the peer firstly performs an IBC setup procedure. The average setup times are about $682.06 \pm$ $404.74 \mathrm{msec}$ and $4789.05 \pm 2203.98 \mathrm{msec}$ for 1024-bit and 2048-bit IBC's, respectively. In Table 2, we measured other step-by-step performance of our prototype implementation of the random visitor scheme for various IBC key sizes. In this measurement, the RSA key size is fixed to 1024 bits. 
Table 2 Timing for IBC operation time in each step. (msec)

\begin{tabular}{cccccccc}
\hline key (bit) & P1 & P2 & P3 & P4 & P5 & P6/P7 & P8 \\
\hline 512 & 31.562 & 11.250 & 7.656 & 15.312 & 7.500 & 4.687 & 3.906 \\
768 & 67.812 & 19.062 & 16.093 & 32.031 & 15.625 & 4.687 & 3.750 \\
1024 & 128.593 & 31.562 & 27.656 & 54.531 & 27.031 & 4.687 & 3.906 \\
1280 & 218.906 & 44.218 & 42.343 & 84.687 & 41.250 & 4.843 & 3.906 \\
1536 & 345.156 & 59.531 & 58.437 & 118.425 & 58.125 & 4.843 & 3.905 \\
2048 & 686.406 & 98.750 & 99.687 & 199.531 & 97.500 & 4.843 & 3.906 \\
\hline
\end{tabular}

Table 3 RSA signature and Triple DES encryption execution time. (msec, message length $=10000$ )

\begin{tabular}{ccc}
\hline & Encryption (Signature verification) & Decryption (Signature) \\
\hline 1024-bit RSA & 4.522 & 5.623 \\
128-bit Triple DES (DES-EDE2) & 2.968 & 3.125 \\
\hline
\end{tabular}

Table 4 Summary of the number of cryptographic operations performed during the identifier verification using random visitor scheme.

\begin{tabular}{llll}
\hline $\begin{array}{l}\text { ID verification trans- } \\
\text { action }\end{array}$ & IBC operations & $\begin{array}{l}\text { Public key op- } \\
\text { erations }\end{array}$ & $\begin{array}{l}\text { Secret key op- } \\
\text { erations }\end{array}$ \\
\hline Initiator & 2 & 1 & 3 \\
Target & 2 & 1 & 3 \\
Delegate & 2 & 0 & 2 \\
\hline
\end{tabular}

Table 5 Size of protocol messages. (byte, 1024-bit IBC)

\begin{tabular}{lllll}
\hline P1 & P2 & P4 & P5 & P7 \\
\hline 692 & 833 & 972 & 972 & 782 \\
\hline
\end{tabular}

We have presented in Table 3 the execution tims for RSA signature and Triple DES encryption implementations on the same machine. The processing times for 1024-bit RSA signature and verification are $5.623 \mathrm{msec}$ and $4.522 \mathrm{msec}$, respectively. For the Triple DES encryption, password-based encryption using DES-EDE2 (128-bit key length) and HMAC/SHA-1 is used. The processing times for Triple DES encryption and decryption are $2.968 \mathrm{msec}$ and $3.125 \mathrm{msec}$, respectively. The signature and encryption times are recorded for a 10,000 byte message.

Table 4 summarizes the number of cryptographic operations performed during a single identifier verification. In our implementation, Triple DES encryption is used instead of IBC encryption. Table 5 presents the number of protocol messages at each transaction. The 1024-bit IBC key length is used for these measurements.

\section{Conclusion and Future Work}

To address the identifier authentication problem (caused by successful identifier forgery attacks such as Sybil and Eclipse) in distributed, serverless P2P networks, we propose the use of trust binding. We proposed a scheme, called random visitor, which combines peer identifiers into cryptographically protected and instantly generated 'moderate trust binding(s)' so that the binding can limit or prevent the possibility of identifier forgery attacks. The scheme is motivated by three main principles: a) blinding an attacker by randomizing the generation of the trust binding, b) hardening identifier security by combining a peer identifier into the trust binding using a cryptographic mechanism called identity-based cryptography (IBC), and c) decreasing the possibility of a successful identifier attack by repeating the scheme.

Analysis results indicate that the scheme can successfully protect against identifier attacks in large sized networks (more than 10,000 peers). For example, if an attacker can forge 100 identifiers out of 10,000 peers, then the possibility of a successful identifier forgery attack is less than 0.02. We implemented the proposed scheme in a Windows platform using a GMP library. From the analysis and measurement results, we conclude that our identifier defense mechanism is strong enough to limit or prevent identifier attacks and is scalable to very large groups with frequent joins and leaves.

Since P2P networks have many dynamic characteristics, however, assessing a security scheme based simply on its functional cost is not sufficient. Therefore, we need a simulation study to evaluate the overall performance of the scheme in a large $\mathrm{P} 2 \mathrm{P}$ network setting.

\section{References}

[1] D. Anderson, "SETI@ @ome," in Peer-to-Peer: Harnessing the Benefit of a Disruptive Technology, ed. A. Oram, O'Reilly \& Associates, Sebastopol, CA, 2001.

[2] S.M. Larson, C.D. Snow, M. Shirts, and V.S. Pande, "FOLDING@home and GENOME@home: Using distributed computing to tackle previously intractable problems in computational biology," Computational Genomics, 2002.

[3] J. Miller, "Jabber: Conversational technologies," in Peer-to-Peer: Harnessing the Benefits of a Disruptive Technology, ed. A. Oram, pp.77-88, O’Reilly \& Associates, Sebastopol, CA, 2001.

[4] Networks.

[5] G. Kan, "Gnutella," in Peer-to-Peer: Harnessing the Benefit of a Disruptive Technology, ed. A. Oram, O'Reilly \& Associates, Sebastopol, CA, 2001.

[6] N. Hemming, "Kazaa," available: www.kazaa.com

[7] C. Shirky, "Listening to napster," in Peer-to-Peer: Harnessing the Benefit of a Disruptive Technology, ed. A. Oram, O’Reilly \& Associates, Sebastopol, CA, 2001.

[8] Skype available: www.skype.com

[9] S.A. Baset and H. Schulzrinne, "An analysis of the skype peertopeer internel telephony protocol," Tech. Rep. CUCS-039-04, Computer Science Department, Columbia University, 2004. 
[10] M. Castro, P. Druschel, A. Ganesh, A. Rowstron, and D.S Wallach, "Secure routing for structured peer-to-peer overlay networks," SIGOPS Oper. Syst. Rev., vol.36, no.SI, pp.299-314, 2002.

[11] J. Douceur, "The sybil attack," Proc. 1st International Peer-To-Peer Systems Workshop (IPTPS), pp.251-260, Cambridge, MA, 2002.

[12] A. Shamir, "Identity-based cryptosystems and signature schemes," Proc. CRYPTO 84 on Advances in Cryptology, pp.47-53, SpringerVerlag New York, Santa Barbara, CA, 1985.

[13] C. Cocks, "An identity based encryption scheme based on quadratic residues," Proc. 8th IMA International Conference on Cryptography and Coding, pp.360-363, Springer-Verlag, 2001

[14] D. Boneh and M. Franklin, "Identity-based encryption from the weil pairing," SIAM J. Comput., vol.32, no.3, pp.586-615, 2003.

[15] L. Martin, "Identity-based encryption: A closer look," ISSA J., pp.22-24, 2005.

[16] G. O'Shea and M. Roe, "Child-proof authentication for MIPv6 (CAM)," SIGCOMM Comput. Commun. Rev., vol.31, no.2, pp.4-8, 2001.

[17] T. Aura, "Cryptographically generated addresses (CGA)," RFC 3972, IETF, 2005.

[18] G. Montenegro and C. Castelluccia, "Statistically unique and cryptographically verifiable (SUCV) identifiers and addresses," Proc Network and Distributed System Security Symposium (NDSS), San Diego, California, USA, 2002.

[19] J. Gu, J. Nah, C. Chae, J. Lee, and J. Jang, "Random visitor: a defense against identity attacks in overlay networks," Proc. 7th International Workshop on Information Security Applications (WISA), pp.601-615, Jeju, Korea, 2006.

[20] S. Ratnasamy, I. Stoica, and S. Shenker, "Routing algorithms for DHTs: Some open questions," Proc. First International Workshop on Peer-to-Peer Systems (IPTPS), ed. P. Druschel, M.F. Kaashoek, and A.I.T. Rowstron, Lecture Notes in Computer Science, vol.2429, pp.45-52, Springer, 2002.

[21] S. Ratnasamy, P. Francis, M. Handley, R. Karp, and S. Schenker, "A scalable content-addressable network," Proc. 2001 conference on Applications, technologies, architectures, and protocols for computer communications, pp.161-172, ACM Press, San Diego, California, United States, 2001.

[22] I. Stoica, R. Morris, D. Liben-Nowell, D.R. Karger, M.F. Kaashoek, F. Dabek, and H. Balakrishnan, "Chord: A scalable peer-to-peer lookup protocol for internet applications," IEEE/ACM Trans. Netw., vol.11, no.1, pp.17-32, 2003.

[23] A. Rowstron and P. Druschel, "Pastry: Scalable, distributed object location and routing for large-scale peer-to-peer systems," Proc. IFIP/ACM International Conference on Distributed Systems Platforms (Middleware), pp.329-350, 2001.

[24] B.Y. Zhao, L. Huang, J. Stribling, S.C. Rhea, A.D. Joseph, and J.D. Kubiatowicz, "Tapestry: A resilient global-scale overlay for service deployment," IEEE J. Sel. Areas Commun., vol.22, no.1, pp.41-53, 2004

[25] L. Gong, "IXTA: A network programming environment," IEEE Internet Computing, vol.5, no.3, pp.88-95, 2001. 613610.

[26] T. Dengler and Others, "JXTA v2.0 protocols specification," Tech. Rep., Sun Microsystems, 2001

[27] L. Ganesh and B.Y. Zhao, "Identity theft protection in structured overlays," Proc. 1st Workshop on Secure Network Protocols (NPSec), Boston, MA, 2005.

[28] T. Condie, V. Kacholia, S. Sank, J.M. Hellerstein, and P. Maniatis, "Induced churn as shelter from routing-table poisoning," Proc. Network and Distributed System Security Symposium (NDSS), 2006.

[29] A. Singh, M. Castro, A. Rowstron, and P. Druschel, "Defending against eclipse attacks on overlay networks," Proc. 11th ACM SIGOPS European Workshop, Leuven, Belgium, 2004.

[30] J. Risson and T. Moors, "Survey of research towards robust peer-topeer networks: Search methods," Tech. Rep. UNSW-EE-P2P-1-1, University of New South Wales, Sept. 2004.

[31] E. Sit and R. Morris, "Security considerations for peer-to-peer dis- tributed hash tables," Proc. 1st International Workshop on Peer-toPeer Systems (IPTPS), 2002.

[32] X. Zeng, R. Bagrodia, and M. Gerla, "GloMoSim: A library for parallel simulation of large-scale wireless networks," Proc. Workshop on Parallel and Distributed Simulation, pp.154-161, 1998.

[33] H. Rowaihy, W. Enck, P. McDaniel, and T.L. Porta, "Limiting sybil attacks in structured peer-to-peer networks," Tech. Rep. NAS-TR0017-2005, Network and Security Research Center, Department of Computer Science and Engineering, Pennsylvania State University, July 2005.

[34] G. Danezis, C. Lesniewski-Laas, M.F. Kaashoek, and R. Anderson, "Sybil-resistant DHT routing," Proc. 10th European Symposium On Research In Computer Security, 2005

[35] J. Dinger and H. Hartenstein, "Defending the sybil attack in p2p networks: Taxonomy, challenges, and a proposal for self-registration," Proc. First International Conference on Availability, Reliability and Security (ARES), pp.756-763, 2006.

[36] S. Capkun, J.P. Hubaux, and L. Buttyán, "Mobility helps peer-topeer security," IEEE Trans. Mobile Computing, vol.5, no.1, pp.43$51,2006$.

[37] E. Okamoto and K. Tanaka, "Key distribution system based on identification information," IEEE J. Sel. Areas Commun., vol.7, no.4, pp. $481-485,1989$.

[38] W. Diffie and M.E. Hellman, "New directions in cryptography," IEEE Trans. Inf. Theory, vol.22, no.6, pp.644-654, 1976.

[39] W. Mao, Modern cryptography: theory and practice, Prentice Hall PTR, Upper Saddle River, NJ, 2004.

[40] M. Mambo, K. Usuda, and E. Okamoto, "Proxy signatures: Delegation of the power to sign messages," IEICE Trans. Fundamentals, vol.E79-A, no.9, pp.1338-1354, Sept. 1996.

[41] F. Dabek, B. Zhao, P. Druschel, J. Kubiatowicz, and I. Stoica, "Towards a common API for structured peer-to-peer overlays," Proc. 2nd International Workshop on Peer-to-Peer Systems (IPTPS), Berkeley, CA, 2003.

[42] K. Aberer, L.O. Alima, A. Ghodsi, S. Girdzijauskas, M. Hauswirth, and S. Haridi, "The essence of P2P: A reference architecture for overlay networks," Proc. 5th IEEE International Conference on Peerto-Peer Computing, Konstanz, Germany, 2005.

[43] K.S. Trivedi, Probability and statistics with reliability, queuing, and computer science applications, 2nd ed., Wiley, New York, 2002.

[44] W. Du, J. Deng, Y.S. Han, P.K. Varshney, J. Katz, and A. Khalili, "A pairwise key predistribution scheme for wireless sensor networks," ACM Trans. Inf. Syst. Secur., vol.8, no.2, pp.228-258, 2005.

[45] H. Chan, A. Perrig, and D. Song, "Random key predistribution schemes for sensor networks," 2003.

[46] Crypto++ Available: http://www.cryptopp.com/

[47] GMP, "GNU Multiple Precision Arithmetic Library," available: http://www.swox.com/gmp

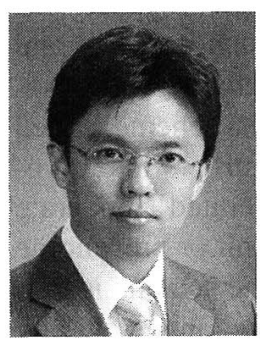

Jabeom Gu received the M.S. and Ph.D. degrees in Electrical Engineering from ChungAng University in 2002 and 2006, respectively. $\mathrm{He}$ is with Electronics and Telecommunications Research Institute, Korea. His research interest includes distributed network security, peerto-peer network, overlay multicasting, wireless network security, and IPTV security. 


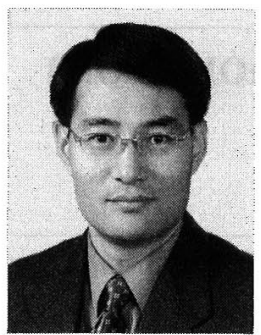

Jaehoon Nah received the M.S. degree in Computer Engineering from Chung-Ang University in 1987. He received the Ph.D. degree in Electronic and Infomation Engineering from Hankuk University of Foreign Studies in 2005. $\mathrm{He}$ is a principal research engineer and a team leader in Division of Information Security in Electronics and Telecommunications Research Institute, Korea. His research interest includes distributed network security, peer-to-pecr nctwork, overlay multicasting, and IPTV security.

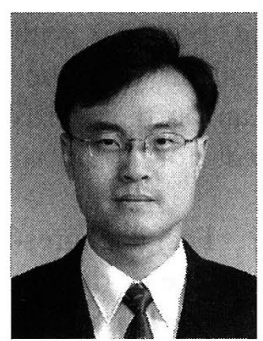

Hyeokchan Kwon received his BS degree in computer engineering from Seowon University in 1994 . He received his $\mathrm{MS}$ and $\mathrm{PhD}$ degrees in computer science from Chungnam National University in 1996 and in 2001 . He joined ETRI in 2001 and has been working on network security. His research interests include network security, P2P, IPv6 and mobile IP security.

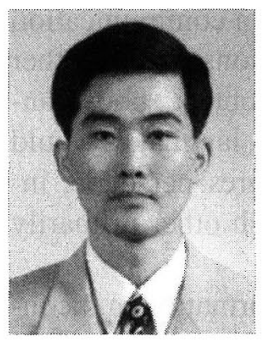

Jongsoo Jang received the B.S and M.S degrees in Electronics Engineering from Kyungpook National University in 1984 and 1986, respectively. He received his $\mathrm{Ph}$. D degree in Computer Engineering from Chungbuk National University in 2000. Since 1989, he has been working with ETRI, Daejeon, Korea and now is the Director of Applied Security Group.

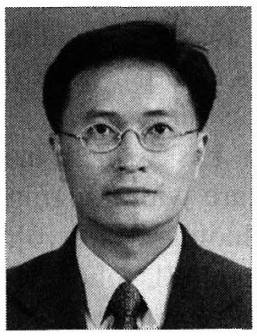

Sehyun Park received the B.S. and M.S degrees in electronics engineering from the Chung-Ang University, Seoul, Korea in 1986 and 1988, respectively, and the Ph.D. from University of Massachusetts, Amherst in 1998. From 1988 to 1999 , he was a senior research staff at ETRI, Korea. He is currently an Associate Professor of School of Electrical and Electronics Engineering at the Chung-Ang University, where he has established the Ubiquitous Computing and Cipher Internet Laboratory. He is the head of Chung-Ang University HNRC (Home Network Research Ccnter)-ITRC (Information Technology Research Center) supported by the MIC (Ministry of Information and Communication), Korea. His major research interests include home networks, ubiquitous computing and network security. 